\title{
Rudolf Schwerdtfeger
}

Im Rahmen dieser Studie wurde Schwerdtfeger schon beiläufig erwähnt. Im vorigen Kapitel wurde z. B. angedeutet, dass er eine sexualisierte Figur von Doktor Faustus ist. $^{1}$ Viele Indizien sprechen dafür: Seine Neigung zum Flirten, seine ehebrecherische Liaison mit Ines Rodde und seine intime Beziehung zu Leverkühn. Schwerdtfeger ist jedoch nicht nur ein anziehender Mann, sondern auch ein talentierter Geigenvirtuose. Der erste Teil des vorliegenden Kapitels beschäftigt sich mit Schwerdtfegers Darstellung im Roman, die dem Mythos des dämonischen Geigers entspricht. Sowohl dieser Mythos, der vor allem in der Form des Paganini-Mythos auftaucht, als auch seine Relevanz in der deutschsprachigen Literatur werden parallel zur Analyse der Figurencharakterisierung des fiktiven Geigers von Doktor Faustus beleuchtet; die Analyse umrahmen Roland Barthes' theoretische Darlegungen zum Mythos und zur Mythenlektüre. Der zweite Teil des Kapitels widmet sich dem dritten Satz von Henzes Violinkonzert, der den Titel „Rudi S.“ trägt. Eher als das Violinkonzert selbst, stehen hier inhaltliche Mikroformen des Romans und die Figurencharakterisierung Schwerdtfegers im Vordergrund: Das Verhältnis der Transposition zur Vorlage lässt sich hier als dezidiert verstärkend definieren. Beide Abschnitte zeigen, um mit Barthes zu argumentieren, dass der Mythos des dämonischen Geigers in vielen Medien immer noch präsent ist, wo er stets neuen Deformationen unterzogen wird.

${ }^{1}$ Vgl. 8.1.2. 


\subsection{Schwerdtfeger in Doktor Faustus}

Die Figur Rudolf Schwerdtfeger ist Teil vieler Kapitel von Doktor Faustus ${ }^{2}$ und ist eine der allerersten Figuren, die Thomas Mann zu Beginn seines Schaffensprozesses am Roman skizziert haben soll: „Figur des Rud. Schwerdtfeger, Geigers aus dem Münchener Zapfenstösser-Orchester ${ }^{* 3}$ heißt es in den Tagebüchern. Die Leser*innenschaft begegnet dem Musiker erst im 23. Kapitel, als Leverkühn nach München umzieht und Schwerdtfeger als Gast des gesellschaftlichen Kreises um die Familie Rodde kennenlernt. Er sei

ein begabter junger Geiger, Mitglied des Zapfenstößer-Orchesters, das neben der Hofkapelle eine bedeutende Rolle im musikalischen Leben der Stadt spielte, und in welchem er unter den ersten Violinen arbeitete. (DF: 290) ${ }^{4}$

In der Forschungsliteratur zum Roman bleibt diese Romanfigur, bis auf wenige Ausnahmen, jedoch relativ unbeachtet. ${ }^{5}$ Seit Beginn seiner Freundschaft $\mathrm{zu}$ Adrian Leverkühn möchte der talentierte Geiger, der nicht nur dem Musizieren, sondern auch - so Zeitblom - ,dem Flirt mit dem schönen Geschlecht, jungen Mädchen sowohl wie reiferen Frauen, selig hingegeben“ (DF: 290) ist, dass der Komponist für ihn ein Violinkonzert schreibt, ,mit dem er sich in der Provinz hören lassen kann“ (DF: 298). Dieses Konzert, das laut der Erzählinstanz ,nicht zu Leverkühns höchsten und stolzesten“ (DF: 573) gehört, wird der Komponist für ihn schließlich komponieren; nicht nur das, Leverkühn wird auch bei allen seinen

\footnotetext{
${ }^{2}$ Ab Kapitel XXII bis zum Kapitel XLII taucht Schwerdtfeger bis auf wenige Ausnahmen (z. B. das Teufelsgespräch) immer auf.

${ }^{3}$ TB1: 29.04.1943, S. 568.

${ }^{4}$ Später wird er die Stelle des Konzertmeisters besetzen: vgl. DF: 506. Laut Dirk Heißerer korrespondiert das von Thomas Mann erfundene Münchener Orchester mit dem KaimOrchester. Vgl. Heißerer, Dirk: Unterwegs im „Lebensbuch“. München in Thomas Manns Roman Doktor Faustus. In: Hirmer, Simone u. Marcel Schellong (Hrsg.): München lesen. Beobachtungen einer erzählten Stadt. Würzburg: Königshausen \& Neumann 2008, S. 109125, hier: S. 117.

${ }^{5}$ Siehe z. B. Riggs, Robert: Violins and Violinists in Literature. In: Ders. (Hrsg.): The Violin. Rochester: University of Rochester Press 2016, S. 36-59 (insb. S. 56 f.); Grimstad, Kirsten J.: The Modern Revival of Gnosticism and Thomas Mann's Doktor Faustus. Rochester/New York: Camden House 2002; Luft, Klaus Peter: Erscheinungsformen des Androgynen bei Thomas Mann. New York (u. a.): Lang 1998, S. 114 ff.; Dill, H. J.: Zur Erklärung des Namens „Pfeiffering“ in Thomas Manns Doktor Faustus. In: Germanic Notes 2 (1971), S. 34 ff.
} 
Aufführungen anders als sonst anwesend sein. ${ }^{6}$ Im Laufe der Arbeit an dem Konzert nimmt die Intimität zwischen Schwerdtfeger und Leverkühn zu: Die beiden umarmen sich, ${ }^{7}$ nennen ,einander Du“ (DF: 603) und nach einer schweizerischen Aufführung des Konzerts erscheinen sie „Hand in Hand“ (DF: 604). Nach der Uraufführung in Wien hatten Geiger und Komponist einige Tage im Schloss Tolna zusammen verbracht, weswegen die Forschungsliteratur unter anderen textuellen Indizien zwischen der Auffassung einer homosexuellen und der einer platonischen Liebe oszilliert - Letzteres scheint u. a. Schwerdtfegers Bezeichnung des Violinkonzerts als ,platonisches Kind“ (DF: 510) zu bestätigen. ${ }^{8}$ Zweifelsohne sind die Romankapitel, die vom Komponieren und der Aufführung des Konzerts handeln, reich an homoerotisch-konnotierten textuellen Hinweisen. Schwerdtfeger ist eine höchst sexualisierte Figur in Doktor Faustus: Mit ihm begeht Ines Rodde - eine der beiden Töchter der Senatorin, bei der Leverkühn in München Schwerdtfeger kennenlernt - kurz nach der Eheschließung mit Dr. Helmut Institoris, ,Ästhetiker und Kunsthistoriker [sowie] Privatdozent an der Technischen Hochschule“ (DF: 417), einen langjährigen Ehebruch. ${ }^{9}$ Ines Institoris soll aber nach Schwerdtfegers Meinung, der dem Komponisten die ehebrecherische Beziehung folgendermaßen schildert, alleine die Schuld tragen:

„Ich kann nichts dafür, Adrian, glaube - glauben Sie mir! Ich habe sie nicht verführt, sondern sie mich, und die Hörner des kleinen Institoris, um diesen dummen Ausdruck zu gebrauchen, sind ausschließlich ihr Werk, nicht meines“. (DF: 508) $)^{10}$

\footnotetext{
${ }^{6}$ Das Violinkonzert wird vom Autor in der Entstehung als „Adrians hybrides Geschenk an die Zutraulichkeit" (Ent: 158) beschrieben.

${ }^{7} \mathrm{Vgl}$. DF: $466 \mathrm{f}$.

${ }^{8}$ Vgl. DF: 574 f. Siehe Grimstad: The Modern Revival of Gnosticism, S. 197. Auch Riggs betrachtet die Beziehung als nicht platonisch. Vgl. Riggs: Violins and Violinists in Literature, S. 57.

${ }^{9}$ Es wurde in der Forschungsliteratur bereits mehrfach hervorgehoben, dass Institoris der Name eines der beiden Verfasser des Malleus Maleficarum ist. Vgl. z. B. Müller, Maria E.: Die Gnadenwahl Satans. Der Rückgriff auf vormoderne Pakttraditionen bei Thomas Mann, Alfred Döblin und Elisabeth Langgässer. In: Röcke, W. (Hrsg.): Thomas Mann. Doktor Faustus 1947-1997. Bern (u. a.): Lang 2001, S. 145-165, hier: S. 155.

${ }^{10} \mathrm{Im}$ selben Zugeständnis betont Schwerdtfeger auch, sie nie geliebt, sondern einfach „,brüderlich-kameradschaftliche Empfindungen für sie“ (DF: 509) gehabt zu haben. Er spricht zudem von einem „Übergewicht der Frau in der Liebe, so, daß er sagen müsse, Ines gehe mit seiner Person, seinem Körper um, wie eigentlich und richtigerweise der Mann umgehe mit dem einer Frau - wozu noch ihre krankhafte und krampfhafte, dabei ganz ungerechtfertigte Eifersucht komme auf den Alleinbesitz seiner Person“ (ebd.).
} 
Von Anfang an ahnt Leverkühn die möglichen, fatalen Konsequenzen einer solchen Beziehung. ${ }^{11}$ Schwerdtfeger findet den Mut, Frau Institoris zu verlassen. Diese kann anfänglich dank des Morphiums, das sie sehr wahrscheinlich von Natalie Knöterich bekommt, das Leben wieder ertragen. ${ }^{12}$

Das Violinkonzert stellt zweifelsohne den Höhepunkt der Beziehung zwischen Leverkühn und Schwerdtfeger dar. ${ }^{13}$ Diese Beziehung findet jedoch gleich nach der Aufführung des Stückes in der Schweiz ihr Ende, da die beiden Marie Godeau kennenlernen. Diese „französische Schweizerin“ (DF: 606) hat „die schönsten schwarzen Augen von der Welt" (DF: 607), teilt Zeitblom seinen Leser*innen mit. Sie sei Zeichnerin und arbeite „für kleinere Pariser Opern- und Singspielbühnen“ (DF: 608). Schwerdtfeger und Leverkühn verlieben sich auf den ersten Blick in sie und Leverkühn will sie nach kurzer Zeit heiraten. ${ }^{14}$ Daher die Idee, den Geiger zu ihr zu schicken, um sie von seinen Gefühlen in Kenntnis zu setzen. Es handelt sich demnach um einen Vorheiratsantrag:

Bringst du mir soviel zurück, daß der Gedanke, mein Leben mit mir zu teilen, ihr nicht ganz und gar zuwider, nicht ungeheuerlich ist, - dann kommt meine Stunde, dann will ich selber mit ihr und ihrem Tantchen reden. (DF: 635 f.) ${ }^{15}$

11 „Übrigens ist das kein Spaß für ihn. - Er soll zusehen, daß er heil aus der Sache davonkommt“ (DF: 435).

${ }^{12}$ Als Ergänzung zu Börnchens Ausführungen über das dritte Kapitel von Doktor Faustus und Esmeralda sei hier am Rande erwähnt, dass Natalia Knöterich auch Esmeralda in Erinnerung ruft, einerseits weil sie „,spanisch-exotisch von Ansehen“ (DF: 475) ist, andererseits weil sie Ines Institoris mit einer Art Gift, nämlich mit Morphium, versorgt. Vgl. Börnchen: Kryptenhall, S. 215.

${ }^{13}$ Vgl. Luft: Erscheinungsformen des Androgynen, S. 115.

${ }^{14}$ Laut Heißerer korrespondiert das Dreieck Leverkühn - Marie - Schwerdtfeger zu dem Thomas Mann - Katia Pringsheim - Paul Ehrenberg. Katia Mann habe die gleichen schwarzen Augen wie Marie gehabt. Siehe Heißerer: Unterwegs im „Lebensbuch“, S. 116. Zu Paul Ehrenberg siehe auch Riggs: Violins and Violinists in Literature, S. 59, Fußnote 9.

${ }^{15}$ Thomas Mann erklärt diesen Plan Leverkühns so: „Adrian schickt den Reinen, Glücklichen, Gewinnenden, teils um durch ihn besser zu werben, teils um ihn zu opfern " (TB2: 25.10.1945, S. 268; Herv. A. O.). Hannum bestätigt und erläutert diese Auffassung: ,[T]he sacrifice is called for outright by Leverkühn, who asks Rudi to propose to Marie Godeau on his behalf". Hannum: Self-Sacrifice, S. 299. Das Motiv von Schwerdtfegers Opferung wird auch in 11.2.2 angesprochen. 
Leverkühns exzentrischer Plan, den Zeitblom heftig kritisiert, ${ }^{16}$ ist zum Scheitern verurteilt und führt nicht zur Verlobung Leverkühns mit Godeau, sondern zu ihrer Verlobung mit Schwerdtfeger. Nach dem letzten Konzert für das Münchner Orchester - der Geiger will nämlich mit seiner Verlobten nach Paris ziehen, wo er die Stelle des Konzertmeisters im „Orchestre Symphonique“ (DF: 646) antreten soll - wird er von einer rachsüchtigen Frau Institoris in einer Tram erschossen. ${ }^{17}$

In diesem Kapitel steht diese Musikerfigur von Doktor Faustus, die mit dem Mythos des dämonischen Geigers in Verbindung gebracht wird, im Zentrum. Als theoretische Untermauerung der folgenden Analyse dienen die Ausführungen von Roland Barthes in Mythen des Alltags.

\subsubsection{Der (alltägliche) Mythos des dämonischen Geigers}

Der Mythos des dämonischen Geigers lässt sich als Modifikation oder mit Barthes als Deformation des ursprünglichen Mythos der Geige als dämonisches Instrument verstehen. $\mathrm{Zu}$ den ersten literarischen und künstlerischen Zeugnissen der Assoziation von Geige und Teufel bzw. auch von Geige und Tod zählen Texte und Manuskriptabbildungen zum Todestanz, die von einem Geige spielenden Tod erzählen oder ihn direkt abbilden sowie Zitate aus Shakespeares Dramen ${ }^{18}$ Dass dieser Mythos, der vor Paganini in der Barockepoche bereits mit den real existierenden Violinisten Tartini und Strunck in Verbindung gebracht wurde, sehr geeignet für die Rezeption im Medium der literarischen Schrift zu sein scheint, mag schon die Beobachtung bestätigen, dass ein aktueller Sammelband zur Violine überraschenderweise nicht mit ihrer Geschichte oder mit Informationen zum Geigenbau, sondern mit dem im Titel einer rhetorischen Frage tragenden Teil „An Instrument oder a Metaphor?" beginnt. ${ }^{19}$ In diesem Teil des Buches wird zunächst die Verbindung von Geige und Tod bzw. von Geige und Teufel in künstlerischen und literarischen Werken ausgelotet, danach wird der Fokus auf Violinist*innen

\footnotetext{
${ }^{16}$ Siehe DF: 642: „Wie bekennt man einer Frau die Liebe eines andern? Neigt man sich zu ihr? Blickt man ihr ins Auge? Nimmt man bittend ihre Hand, die man gern in die des Dritten legen zu wollen erklärt?““

${ }^{17}$ Vgl. DF: Kap. XLII.

${ }^{18}$ Vgl. Berger, Robert W.: The Devil, the Violin, and Paganini: The Myth of the Violin as Satan's Instrument. In: Religion and the Arts 16 (2012), S. 305-327, hier: S. 309; Shakespeare, W.: Henry VIII. In: Ders.: The Complete Works, Part 2, Akt 1 Szene 3, 714-752, hier: S. 721; Riggs, R.: Association with Death and the Devil. In: Ders. (Hrsg.): The Violin, S. 3-35.

${ }^{19}$ Riggs (Hrsg.): The Violin, S. vii.
} 
und das Instrument in der Literatur gelegt, wobei die meisten Geiger*innenfiguren dämonische Züge tragen und so auch Schwerdtfeger aus Thomas Manns Roman selbstverständlich nicht fehlt. ${ }^{20}$ In der vorliegenden Studie wird nicht wie bei Riggs von Metaphern, sondern von Mythen gesprochen, denn speziell die Verflechtung mit real existierten Musikern wie Paganini oder Tartini macht dieses zweite semiologische System sichtbar, das auf einem Signifikanten beruht, der zugleich Sinn und Form ist. Nicht zufällig weist Barthes darauf hin, dass ein Mythos wohl deformieren kann, jedoch nichts verbirgt: ${ }^{21}$ Obwohl real existierte Musiker vom Mythos deformiert werden können, bleiben die Vorlagen erkennbar. Die Bezeichnung ,Metapher' scheint daher für ein Phänomen, das Fakten und Persönlichkeiten des Musiklebens sowie u. a. literarische und anekdotische Texte über die Epochen hinweg einschließt und deformiert, unzureichend.

Laut Riggs ist Schwerdtfeger ,a demonic character“22 und auch Zeitblom spricht im Roman von seiner „kindischen Dämonie“ (DF: 604): Sein Talent für den Flirt und das Geigenspiel verbindet sich mit einer gewissen Naivität, was der Erzähler einem dämonischen Ursprung zuschreibt. Inwieweit sich Schwerdtfeger, der wohl auch für eine Opferfigur von Doktor Faustus gehalten werden kann, als Verkörperung des Mythos des dämonischen Geigers auffassen lässt, sei im Folgenden aufgezeigt. Bei der Erwähnung seines Repertoires taucht Paganini nie auf, Tartini wird jedoch explizit benannt. ${ }^{23}$ Des Weiteren spielt Schwerdtfeger das typische Repertoire eines Geigenvirtuosen, etwa Bachs Partita in E-Dur, Vivaldi, Spohr, Vieuxtemps, Grieg, Beethovens Kreutzer-Sonate und Dvořáks Humoreske. ${ }^{24}$ Dass auf Tartini explizit verwiesen wird, spricht auch wieder für jene Kontamination des Romans durch das System der Barockmusik, mit dem sich der Viola d'amore-Spieler Zeitblom gut auskennt; ${ }^{25}$ dass hingegen Paganini namentlich nicht erwähnt wird, ist in Bezug auf Doktor Faustus, wo etwa die Namen

\footnotetext{
${ }^{20}$ Vgl. Riggs: Association with Death and the Devil u. Ders.: Violins and Violinists in Literature.

${ }^{21}$ Barthes: Mythen des Alltags, S. 268 u. 277.

${ }^{22}$ Riggs: Violins and Violinists in Literature, S. 57.

${ }^{23}$ Siehe DF: 506 u. 291.

${ }^{24}$ Vgl. DF: 506 u. 622; zur Geschichte und zum Repertoire des Instruments: Nardolillo, Jo: The Canon of Violin Literature: a Performer's Resource. Lanham, Md. (u. a.): Scarecrow Press 2011; Menuhin, Yehudi: The Violin. Paris: Flammarion 1996.

${ }^{25}$ Vgl. 11.1.2. In Bergers Aufsatz wird außerdem eine Anekdote erwähnt, die ein Treffen Struncks mit Corelli betrifft und das virtuose Spielen mittels Anwendung der Scordatura ebenfalls mit dem Dämonischen verknüpft. Vgl. Berger: The Devil, the Violin, and Paganini, S. 313. Hier sei zudem erwähnt, dass auch Paganini auf die Scordatura zurückgegriffen haben soll. Vgl. Grisley, Roberto: Art. Paganini, Nicolò, Würdigung. In: MGG
} 
Schönberg oder Nietzsche nie auftauchen, kein Beweis dafür, dass der Mythos des dämonischen Geigers hier ausnahmsweise nicht auf den Paganini-Mythos zurückgreift. ${ }^{26}$ Einzelheiten aus Anekdoten über den italienischen Violinisten, Autor der Ventiquattro Capricci für Violine solo, die als Meilenstein des Geigenvirtuosentums gelten, finden im 19. Jahrhundert Eingang u. a. in Texte von Grillparzer und Heine. In vielen literarischen und nicht-literarischen Texten stellt Paganini aufgrund seiner Biographie, die im Folgenden dargelegt wird und seines meisterhaften, virtuosen Geigenspiels die Verkörperung par excellence des Mythos des dämonischen Geigers dar. ${ }^{27}$

Auch die folgende Analyse stützt sich vor allem auf den Paganini-Mythos, um die Darstellung von Schwerdtfeger als Paganini von Doktor Faustus zu beleuchten. Der italienische Geiger sei Anekdoten der Zeit zufolge nicht nur für sein unerreichbar virtuoses Geigenspiel, sondern auch für seine Fähigkeit berühmt gewesen, Tiergeräusche, das Seufzen und Stöhnen von Liebenden und das Weinen alter Frauen zu imitieren. ${ }^{28}$ Schwerdtfeger kann ebenfalls das traditionelle Violinrepertoire meisterhaft spielen und zudem hervorragend pfeifen:

Ich [Zeitblom] habe das später auch bei Roddes und Schlaginhaufens gehört und mir von ihm erzählen lassen, wie er schon als ganz kleiner Junge, bevor er Violinunterricht bekam, diese Technik auszubilden begonnen und sich im reinen Nachpfeifen vernommener Musikstücke, fast wo er ging und stand, geübt, auch später an dem Erworbenen immer fortentwickelt hatte. Es war glänzend, - eine kabarettreife Fertigkeit, die fast mehr imponierte als sein Geigenspiel, und für die er organisch besonders glücklich angelegt sein mußte. (DF: 380)

Dieses Talent von Schwerdtfeger ist so ausgeprägt, dass Zeitblom ihn nach dieser ersten Erwähnung immer häufiger als „Geiger und Pfeifer“ (DF: 401) anredet. Auch das Pfeifen lässt sich mit Paganini in Verbindung bringen, und zwar mit seinem Imitationstalent, denn durch das Pfeifen ahmt der Geiger musikalische Kompositionen, die ursprünglich etwa für Violine, Flöte und Harfe geschrieben wurden, nach. ${ }^{29}$

Online. Zuerst veröffentlicht 2004, online veröffentlicht 2016.<https://www.mgg-online. com/mgg/stable/49503 > (letzter Zugriff: 21.08.2020) u. Kap. 7.

${ }^{26} \mathrm{Vgl}$. 8.1.

${ }^{27}$ Vgl. Cersowsky, Peter: „Mehr als Musik“. Paganini in der deutschen Literatur seiner Zeit. In: Archiv für das Studium der Neueren Sprachen und Literaturen 156 (2004) H. 1, S. 157-167.

${ }^{28}$ Siehe Berger: The Devil, the Violin, and Paganini, S. 318.

${ }^{29}$ Vgl. DF: $507 \mathrm{f}$. Wahrscheinlich ist es auch kein zufälliges Textelement, dass zu den Gästen der Familie Institoris auch ein Maler gehört, der ,für die lustige Imitation von 
Verschiedene Skandalgeschichten verknüpfen sich außerdem mit Paganini: Manche Anekdoten und literarische Texte, z. B. von Grillparzer und Heine, erzählen von einem Mord an einer Liebhaberin, manche von der Vergewaltigung einer Minderjährigen; darüber hinaus soll Paganini in einem Friedhof konzertiert haben, was ihm später verboten wurde. Nach seinem Tod in Nizza soll dort aufgrund des Vergewaltigungsskandals seine kirchliche Bestattung nicht erlaubt gewesen sein. ${ }^{30}$ Die Assoziation mit dem Teufel lässt sich u. a. einer Anekdote der Zeit entnehmen, von der Paganini selbst in der Revue Musicale berichtet: Ein Zuschauer soll bei einer Aufführung den Teufel gesehen haben, der seinen Bogen leitete. ${ }^{31}$ Dies lässt zugleich an die Anekdote über Tartinis Sonate Le trille du diable, da der Teufel dem Geiger im Traum die Sonate diktiert haben soll, ${ }^{32}$ und an Shakespeare denken: Hier diktiert der Teufel keine Sonate, leitet aber selbst Paganinis Bogen. Dies alles sind Adaptionen ein und desselben Mythos, der speziell den Bogen als Teufelswaffe ansieht. Schenkt man dem Nachnamen der Geigerfigur von Doktor Faustus Aufmerksamkeit, so wird man feststellen, dass dieser aus zwei Wörtern besteht: Das erste Wort, ,Schwerdt', bezeichnet eine Waffe, die optisch nicht zuletzt auch aufgrund der Präsenz eines Handgriffs einem Bogen ähnelt. Das zweite Wort, ,Feger', kann auf einen - so im Duden - „freche[n] Bursche[n]“33 bzw. auf einen - so im Schweizerischen Idiotikon - ,tüchtige[n] Kämpfer“34 bzw.

Schauspielern, Tieren, Musikinstrumenten und Professoren“ (DF: 477) sehr begabt ist. Obwohl sich dies auf eine andere Figur bezieht, verstärkt es indirekt den Bezug auf den Paganini-Mythos, da Schwerdtfeger Liebhaber von Ines Institoris ist und wenige Seiten später vom Ehebruch erzählt wird. Zu Schwerdtfegers Pfeifen vgl. auch: Dill: Zur Erklärung des Namens „Pfeiffering“.

${ }^{30}$ Vgl. Berger: The Devil, the Violin, and Paganini, S. 319 f.; Cersowsky: „Mehr als Musik“, S. 158 u. 162.

${ }^{31}$ Zit. in Berger: The Devil, the Violin, and Paganini, S. 322.

${ }^{32}$ Siehe ebd., S. 314 f.

${ }^{33}$ „Feger“. In: Duden Online.<https://www.duden.de/rechtschreibung/Feger $>$ (letzter Zugriff: 21.08.2020).

34 „Feger“. In: Schweizerisches Idiotikon.<https://digital.idiotikon.ch/idtkn/id1.htm\#!page/ 10685/mode/1up >(letzter Zugriff: 21.08.2020). Des Weiteren könnte das Kompositum ,Schwerdtfeger' bzw. ,Schwertfeger" auf die handwerkliche Qualität des Spielens hinweisen und daher die mittelalterliche Einteilung von Musiker*innen in musici theorici und musici practici in Erinnerung rufen. Ersteres lässt sich auf Leverkühn als Komponisten und Kompositionstheoretiker, Letzteres auf Schwerdtfeger als Geigenvirtuosen übertragen. Siehe auch „Schwertfeger“. In: Grimm, Jacob u. Wilhelm: Deutsches Wörterbuch, Bd. 15 Sp. 2587 bis 2588.<https://www.woerterbuchnetz.de/cgi-bin/WBNetz/wbgui_py?sigle= DWB\&lemid=GS22166 > (letzter Zugriff: 21.08.2020) u. Salmen, Walter: Art. Musiker, Zum Terminus. In: MGG Online. Veröffentlicht November 2016.<https://www.mgg-onl ine.com/mgg/stable/12883 > (letzter Zugriff: 21.08.2020). 
„starke[n] Mann“35 hinweisen, was Schwerdtfeger zweifelsohne ist. Zeitblom beschreibt ihn als ,eifrige[n] Salonbesucher, der jeden freien Abend in mindestens einer, meistens aber zwei bis drei Gesellschaften verbrachte" (DF: 290) und sich ,dem Flirt mit dem schönen Geschlecht, jungen Mädchen sowohl wie reiferen Frauen, selig hingegeben“ (ebd.) hatte. Nicht nur, Schwerdtfeger macht auch Leverkühn den Hof und ist vergleichbar zur Darstellung Paganinis in diversen Texten sowohl bei Frauen als auch bei Männern beliebt. ${ }^{36}$ Was Schwerdtfegers Skandalgeschichten angeht, so seien hier nochmals - um nur einige Beispiele zu nennen - die ehebrecherische Liaison mit Ines Institoris und die homosexuelle Beziehung zu Leverkühn erwähnt. Wichtige Modifikation des Mythos stellt die Tatsache dar, dass Schwerdtfeger selbst ermordet wird, was sich als umgekehrte Version des Paganini-Mythos lesen lässt. Die Verbindung von Geige und Tod ist aber somit gegeben; nicht zufällig erwähnt Zeitblom, dass Schwerdtfeger in der Tram „seinen Geigenkasten aufgestellt zwischen den Knien“ (DF: 649) sitzt.

Erstaunlicherweise wird in Anekdoten und sonstigen Texten zu Paganini eher als das Spielen das Aussehen des Geigers detailliert beschrieben, weswegen Cersowsky von einem „Vorrang des Optischen“37 ${ }^{\text {spricht. }}{ }^{38}$ Zeitbloms Darstellung lässt sich zwar in dieser Hinsicht als ausgewogener werten, da auch sein Spielen und Pfeifen ausführlich geschildert werden. Er verzichtet jedoch auch nicht darauf, seine Leser*innenschaft wiederholt über die Kleidung des Geigers zu informieren: So erscheint Schwerdtfeger in jenen Münchner Abendkreisen ,als Bauernbursch gekleidet oder in der Tracht des florentinischen 15. Jahrhunderts, die seinen hübschen Beinen zustatten kam und ihn Botticellis Jünglingsportrait mit der roten Mütze nicht unähnlich machte“ (DF: 296). ${ }^{39}$

Hinsichtlich eines Vergleichs der Umstände des Todes der beiden Geiger sei Folgendes erwähnt: Paganini stirbt in Nizza und soll nach seinem letzten Konzert in München mit Lorbeer gekrönt worden sein ${ }^{40}$ Schwerdtfeger will ebenfalls nach seinem letzten Konzert in München nach Frankreich, und zwar nach Paris umziehen, wird jedoch kurz nach dem Konzert von Ines Institoris ermordet.

\footnotetext{
${ }^{35}$ Ebd.

${ }^{36}$ Vgl. DF: 291 f.; Cersowsky: „Mehr als Musik“, S. 160 u. 166.

${ }^{37}$ Cersowsky: „Mehr als Musik“, S. 166.

${ }^{38}$ Vgl. auch Berger: The Devil, the Violin, and Paganini, S. 323.

${ }^{39}$ Und (wieder vermutlich nicht zufällig) liegt Florenz in derselben Region wie Lucca, der Geburtsstadt Paganinis. Darüber hinaus verknüpft die rote Mütze Schwerdtfeger mit dem „teuflischen“ Leipziger Dienstmann. Vgl. 6.1.1.

${ }^{40}$ Zit. in Cersowsky: „Mehr als Musik“, S. 164.
} 
Der bisher geschilderte Vergleich zwischen Schwerdtfeger und Paganini verfolgt keine hermeneutisch-positivistischen Zwecke, sondern nimmt auf den durch Texte und Bilder verschiedener Art verbreiteten Paganini-Mythos Bezug, der als Subkategorie des Mythos des dämonischen Geigers gilt, der selbst der Assoziation von Geige und Teufel entspringt. Roland Barthes zufolge kann ,,alles Mythos werden, was in einen Diskurs eingeht [...], denn das Universum ist unendlich suggestiv“" ${ }^{41}$ Gleichzeitig gebe es aber keine ewigen Mythen, denn die Geschichte allein „,bestimmt über Leben und Tod der mythischen Sprache“. ${ }^{42}$ Der Mythos umfasse außerdem, ,zwei semiologische Systeme, von denen das eine gegenüber dem anderen ausschert“ ${ }^{43}$ eine ,Objektsprache‘, ,deren sich der Mythos bemächtigt, um sein eigenes System zu konstruieren“44 und eine ,Metasprache', , in der man von der ersten spricht" ${ }^{45}$ Grundlegendes Merkmal des mythischen Begriffs besteht für Barthes darin, „,angepaßt zu sein““46 „Ihm steht eine unbegrenzte Menge von Signifikanten zur Verfügung““. ${ }^{47}$ Dies bestätigt die Beobachtung der vielen Signifikanten des Mythos des dämonischen Geigers (Tartini, Paganini, Schwerdtfeger): Auch hier bemächtigt sich die mythische Sprache durch ,eine vorübergehende kleine Dieberei“"48 der Objektsprache, z. B. der Biographien von real existierten Geigern, um sie dann in nicht identischer Form beispielsweise durch Anekdoten, Gedichte und Romane zurückzugeben. ${ }^{49}$ Nichtsdestotrotz verbirgt aber der Mythos, wie bereits antizipiert, nichts und lässt etwa in Bezug auf Doktor Faustus Anekdoten über Tartini, Strunck, Paganini sowie auch ihre Biographien hinter Zeitbloms Darstellung der Figur Schwerdtfeger erscheinen; zu Recht ist Christine Lubkoll der Auffassung, dass die Thematisierung von Musiker*innen in der Literatur ,als Projektionsfläche“50 dienen und ,zum Mythos narrativiert“51 werden könne. Dass der Mythos des dämonischen Geigers immer

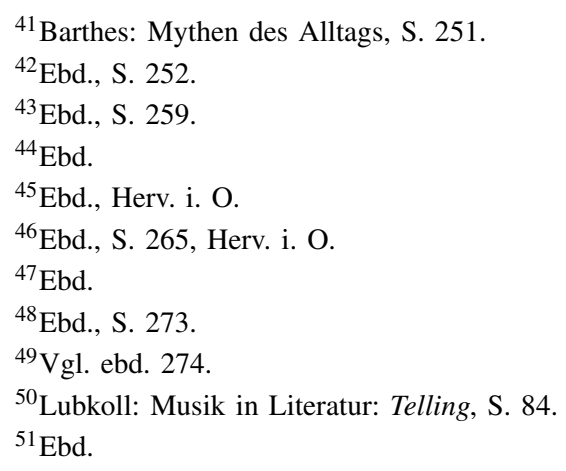


noch aktuell ist, bestätigen etwa journalistische Darstellungen von heutigen Geigenvirtuos*innen, die von Teufelsgeigern reden und deren Aussehen ins Zentrum rücken. ${ }^{52}$

\subsection{Vom Roman zur Musik}

Der vorliegende Abschnitt widmet sich dem letzten Satz von Henzes Violinkonzert, der sich zugleich als partielle intermediale Transposition des fiktiven Konzerts und als Darstellung mit musikalischen Mitteln einer Romanfigur und der mit ihr verbundenen Kapitel begreifen lässt. Auch Henzes Violinkonzert setzt sich mit dem Mythos des dämonischen Geigers auseinander.

\subsubsection{Möglichkeiten der Verstärkung einer literarischen Vorlage: Henzes Rudi S.}

Der dritte Satz des im vorigen Kapitel bereits betrachteten Violinkonzerts von H. W. Henze ist dem Geiger Rudolf Schwerdtfeger gewidmet. Die erste Frage, die spontan in Bezug auf den Titel gestellt werden könnte, ist: Warum verwendet Henze den abgekürzten Namen als Titel des Satzes? Timo Sorg behauptet, die Identität Schwerdtfegers werde auf diese Weise geschützt, ,wie etwa in einem Zeitungsbericht“"53 Die Musik behält tatsächlich die Spannung sowie den Krimi-Charakter der Schwerdtfeger-Kapitel von Doktor Faustus bei. „Ein anderer Grund“, 54 fügt der Musikwissenschaftler hinzu, ,könnte im vertrauten Verhältnis zwischen Rudi und Leverkühn liegen“.55 Die Komposition spricht aus diesem Grund direkt im Titel Leverkühns enges Verhältnis zu Schwerdtfeger an, darauf weist auch die anfängliche Angabe auf den Noten der Violine solo - ,,canto dolcissimo“ (VK: 45, T. 2), ,sehr süßer Gesang“ - hin. ${ }^{56}$ Diese Angabe verdeutlicht zudem, dass sich hier die Instrumentalkomposition dem Gesang annähern möchte.

\footnotetext{
${ }^{52}$ Vgl. z. B. zu David Garrett: Krause, Tilman: Teufelsgeiger müssen einfach sexy sein. In: Welt, 08.06.2016.<https://www.welt.de/kultur/article156072126/Teufelsgeigermuessen-einfach-sexy-sein.html > (letzter Zugriff: 21.08.2020).

${ }^{53}$ Sorg: Beziehungszauber, S. 281.

${ }^{54}$ Ebd.

${ }^{55}$ Ebd.

${ }^{56}$ Jens Rosteck nennt außerdem ein biographisches Ereignis: Ein im Gegensatz zu Doktor Faustus nicht tödliches Attentat auf einen Freund Henzes, der ebenfalls Rudi hieß. Siehe Rosteck: Hans Werner Henze, S. 319.
} 
Die zweite Frage, die sich die Leser*innen von Doktor Faustus stellen könnten, hat mit der Reihenfolge der Konzertsätze zu tun: Der zweite Satz ist Echo gewidmet, aber sein Ankommen in Pfeiffering findet nach dem Tod des Geigers statt. Schmierer begründet diese Entscheidung so: „Er [der dritte Satz] ist jedoch als nochmals übersteigerter Schicksalsschlag in doppeltem Sinne auskomponiert und steht deshalb am Schluss". ${ }^{77}$

In Bezug auf die Vorlage folgt daher die intermediale Transposition einer anderen „Anordnung der Ereignisse oder zeitlichen Segmente“. ${ }^{58}$ Henzes dritter Satz stellt nicht nur einen Versuch dar, im Medium der instrumentalen Musik die Figur Schwerdtfegers zu charakterisieren, sondern lässt sich auch als Transposition des Violinkonzerts begreifen und hätte wohl auch dem zweiten Teil der vorliegenden Studie, der sich mit einigen fiktiven Kompositionen Leverkühns befasst, zugeordnet werden können. Zeitbloms Darstellung des Musikstils des Werkes scheint mit dem Gesamtstil von Henzes Kompositionen, den der Autor als musica impura bezeichnet, ${ }^{59}$ gut vereinbar zu sein: Die Erzählinstanz beschreibt Leverkühns Komposition als eine, die ,durch eine gewisse verbindliche virtuos-konzertante Willfährigkeit der musikalischen Haltung ein wenig aus dem Rahmen von Leverkühns unerbittlich radikalem und zugeständnislosem Gesamtwerk" (DF: 592 f.) fällt. Leverkühn selbst erkennt eine gewisse, in seinem Schaffen selten vorhandene „Menschlichkeit“ in seinem Violinkonzert, das er einer Person gewidmet habe, bei der er „zum erstenmal in [s]einem Leben menschliche Wärme fand“ (DF: 633). Diese durch das Violinkonzert vermittelte „Menschlichkeit“ spricht Henzes Satz im paratextuellen Hinweis des Titels sofort an, der zudem darauf aufmerksam macht, dass Schwerdtfeger Leverkühn ,zum Du bekehrte“ (ebd.). Im Medium der instrumentalen Musik, wo eine gewisse Vagheit des Erzählens herrscht, lässt sich die Frage, wie die Art der Beziehung zwischen Leverkühn und Schwerdtfeger dargestellt wird, anhand viel weniger Indizien als in einem Erzähltext beantworten. Der Titel und die Angabe „canto dolcissimo“, welche die intime Beziehung zwischen Leverkühn und Schwerdtfeger in den Mittelpunkt rücken, deuten auf ein mehr als professionelles Verhältnis hin, das also weit über die Zusammenarbeit für die Entstehung des Violinkonzerts geht. ${ }^{60}$

\footnotetext{
${ }^{57}$ Schmierer: Musik als Sprache, S. 299.

${ }^{58}$ Genette: Die Erzählung, S. 22.

${ }^{59}$ Vgl. Schmidt, Stephan Sebastian: Opera impura. Formen engagierter Oper in England. Trier: WVT 2002, S. 243-246, hier: S. 243 (Interview des Autors mit Henze).

${ }^{60}$ Wie Manzoni war Henze zeit seines Lebens auch sehr politisch engagiert; die Thematik der Homosexualität lag ihm besonders am Herzen: „Ich denunziere die bürgerliche Gesellschaft und unter anderem, nebenbei bemerkt, auch die Leute, Spießer z. B., die sich über die Schwulen mokieren. Da gibt es doch häufig so einen gewaltsamen Hohn.
} 
Der Satz (teil-)reproduziert zwei Mikroformen: Zum einen Schwerdtfegers Darstellung im Roman durch Zeitblom, sprich: die Figurencharakterisierung durch telling und showing der Erzählinstanz, und zum anderen seine Beschreibung des fiktiven Violinkonzerts, sprich: das dieses Musikwerk betreffende intermediale telling. Zum einen bietet der Satz der Violine solo ,große Gelegenheit zum ,Flirt““ (DF: 594) wie das Konzert Leverkühns: ${ }^{61}$ Es gibt viele virtuose Passagen, die wie ein „,canto dolcissimo“ (VK: 45), ,con grazia“ (VK: 49 und 51), also ,,anmutig“, oder „con bravura“ (VK: 57), also „mit Bravur“ zu spielen sind sowie eine Kadenz, die etwa zwei Minuten dauert und daher einen wichtigen Teil der Komposition darstellt. ${ }^{62}$ Nicht nur an dem Virtuosentum à la Paganini, sondern auch an der ,,an der Grenze des Spottes gehaltenen Süße und Zärtlichkeit“ (DF: 593) von Leverkühns Werk scheint sich Henzes Satz zu orientieren. Die Kadenz, der Inbegriff des Virtuosentums, beinhaltet auch melancholische Töne. Andere Passagen des Satzes wirken zugleich spielerisch und bedrohlich, lassen das dramatische Schicksal Schwerdtfegers erahnen. Henzes dritter Satz versucht, Zeitbloms Erzählweise zu simulieren, die immer wieder den Tod des Geigers antizipiert. ${ }^{63}$ So Schmierer: ${ }^{64}$

Die Tragik kündigt sich [...] bereits nach den ersten Takten an: Der ,Canto dolcissimo' auf sattem Streicherklang wird durch pochende, hintergründige Holzbläserakkorde gestört, die kantable Linie zerfällt, die Schläge der großen Trommel und der Blechbläserklang kündigten den schicksalhaften Schlag an, der am Schluss des Satzes durch knallende Peitschenklänge unterstrichen wird - die Schüsse aus dem Revolver wiedergebend.

Die Musik wird tatsächlich immer erregter und der Peitschenklang bringt die Sologeige zum Schweigen, was innerhalb der Handlungslogik der instrumentalen Komposition zu bedeuten scheint, dass Schwerdtfeger an dieser Stelle seine Seele aushaucht (Abbildung 9.1) :

Ich wehre mich schon mit meiner ersten Note gegen solchen eventuellen Hohn“. Ebd., S. 245. Sorg interpretiert das Violinkonzert Henzes als „Deutschlandbild“: In dieser Hinsicht stehe Rudi symbolisch für die Situation der Homosexuellen im Dritten Reich. Vgl. Sorg: Beziehungszauber, S. 303.

${ }^{61}$ Vgl. auch Sorg: Beziehungszauber, S. 284 f.

${ }^{62}$ Der ganze Satz dauert ca. sieben Minuten.

${ }^{63}$ Siehe z. B. DF: 574 f.: „Nicht ich war ihm dabei zur Seite und hätt'es nicht sein können, selbst wenn er mich dazu aufgefordert hätte. Schwerdtfeger war es, er könnte berichten. Aber er ist tot. - "

${ }^{64}$ Schmierer: Musik als Sprache, S. 299. 


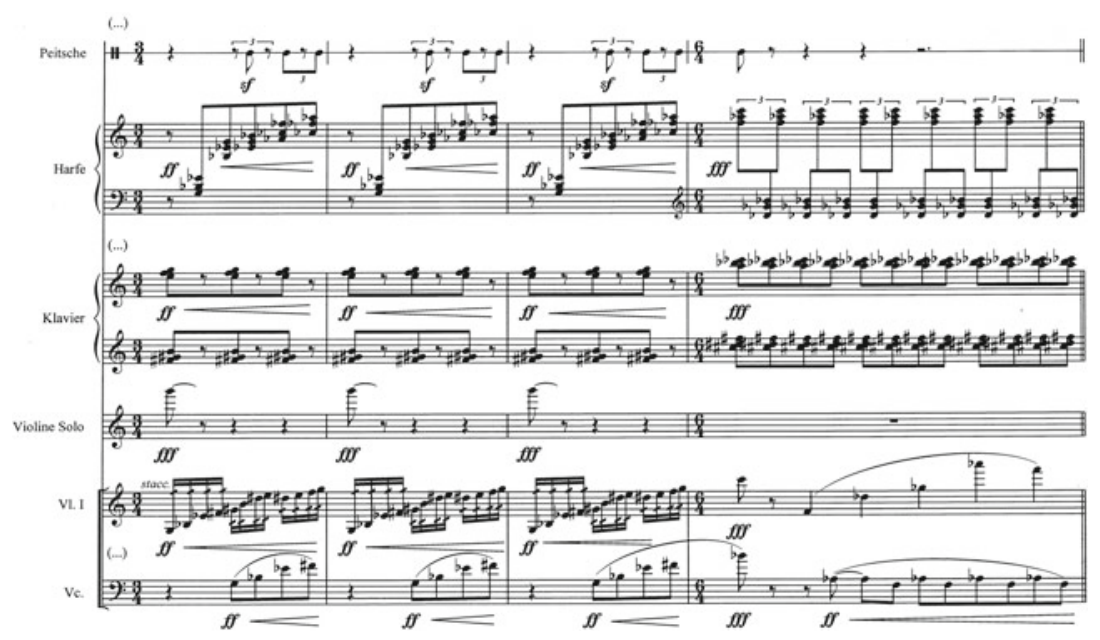

Abbildung 9.1 Der Tod Rudis (VK: 62) (Das Zeichen (...) bedeutet, dass nur eine Auswahl der Orchesterbesetzung wiedergegeben wird.)

Die Violine solo ist nach den kurzen, geseufzten Noten im Sforzato, die man in der Abbildung sieht, nicht mehr zu hören, jedoch zwei Mal noch die Peitsche, die für die wiederholten Schüsse durch Ines steht. ${ }^{65}$ Und dann das Tamtam, das laut Sorg ,idiofone[] Klangsymbol des Todes“. 66

Der Satz schließt mit einer kurzen, akzentuierten Note im ffff des ganzen Orchesters (Abbildung 9.2) :

Die instrumentale Komposition ist in der Lage sowohl die Spannung als auch die inhaltlichen Aspekte der Schwerdtfeger-Kapitel partiell zu reproduzieren, indem sie etwa Erzählstrategien und sonstige Mittel durch bestimmte Musikinstrumente, Rhythmen und Dynamiken ersetzt, die gewisse Klangeffekte erzielen. Diese Elemente rufen zusammen mit den paratextuellen Angaben die Episode in Erinnerung; die Spannung bleibt bis zum letzten, im vierfachen Forte zu spielenden Ton aufrecht erhalten, nur der Applaus des Publikums kann hier die Illusion durchbrechen.

Was die Transposition von Leverkühns Violinkonzert angeht, lässt sich sagen, dass Henzes dritter Satz zum großen Teil einen eigenen Weg geht: Er versucht

${ }^{65} \mathrm{Vgl}$. DF: 650.

${ }^{66}$ Sorg: Beziehungszauber, S. 286. 


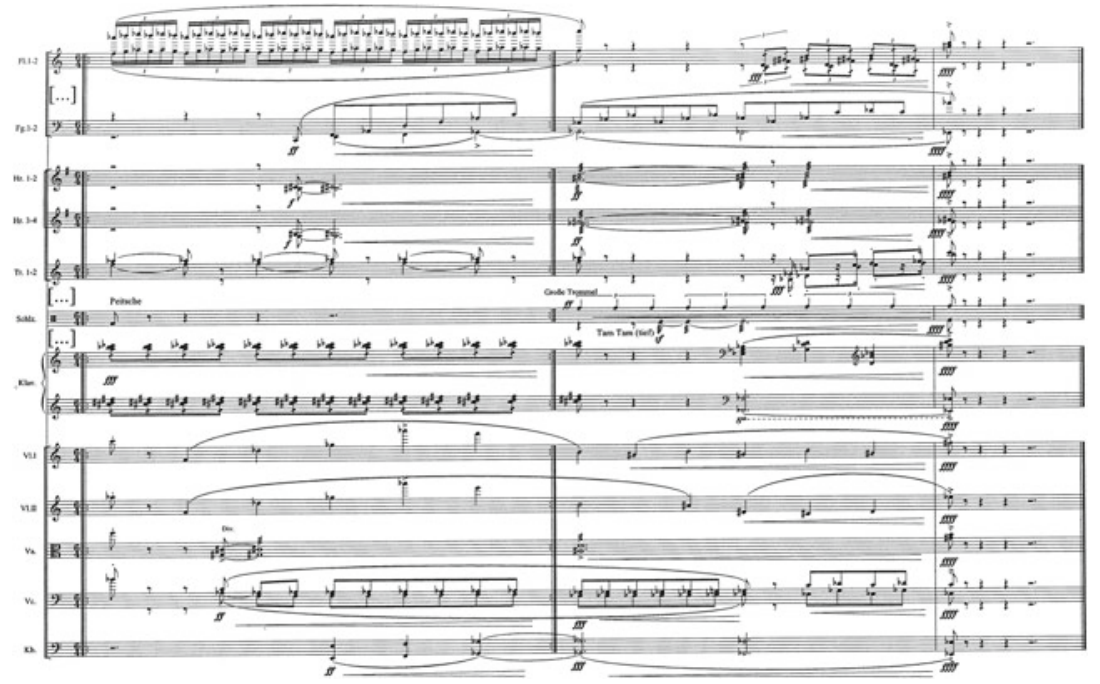

Abbildung 9.2 Das Ende des Satzes (VK: 63 f.)

nicht, die dreiteilige Struktur des fiktiven Werkes zu reproduzieren, das sich aus einem „Andante amoroso“ (DF: 593), einem virtuosen, ,ein Zitat aus Tartinis Teufelstriller-Sonate“ (DF: 594) enthaltenden Scherzo und einem dritten Satz mit Variationen zusammensetzt, obwohl sich vereinzelte Eigenschaften wiederfinden lassen. Anders als die anderen Sätzen gibt es hier nur ein Andante (abgesehen selbstverständlich von der frei zu spielenden Kadenz). ${ }^{67}$ Dementsprechend ist die Einordnung von Henzes Satz in dieses Kapitel durchaus angemessen, weil das, was er vor allem (teil-)reproduziert, inhaltliche Substrate des Romans sind, z. B. Eigenschaften von Schwerdtfeger oder seinem Geigenspiel, seine Aufführung von Leverkühns Violinkonzert, seine Beziehung $\mathrm{zu}$ ihm sowie seine Ermordung in München. Das ausgeprägte Virtuosentum des Satzes ermöglicht ebenfalls eine Zuordnung Schwerdtfegers zum Paganini-Mythos, nicht zuletzt, weil der Musikstil auch auf den von Paganinis Violinkonzerten verweist.

Die Betrachtung des dritten Satzes von Henzes Violinkonzert macht außerdem den Nutzen einer Studie, die auch mit werkexterner Intermedialität arbeitet, sichtbar: Eine in der Forschungsliteratur sonst relativ vernachlässigte Romanfigur gewinnt in der Transposition an Bedeutung und bietet den Anlass zu

${ }^{67}$ Vgl. VK: 45 u. 54. 
neuen Interpretationen eines bereits weit rezipierten literarischen Werkes. Dass Henzes Konzert auf diesen letzten Satz abzielt, mag sowohl die Position des Schwerdtfeger gewidmeten Satzes als auch die Form des Werkes und die Wahl des solistischen Instruments bestätigen, das eben ein Violinkonzert wie das für Schwerdtfeger von Leverkühn geschriebene ist. Um im Forschungsparadigma der Intermedialität einen Schritt weiter zu gehen und zugleich ein Fazit aus dieser Analyse zu ziehen, lässt sich sagen, dass der intermediale Effekt der Verstärkung einer skalaren Auffassung bedarf. Geht man dementsprechend von Graden der Verstärkung von Mikroformen einer literarischen Vorlage durch Medienwechsel bzw. Medientransformation aus, sprich: untersucht man, wie schwach oder wie stark etwa bestimmte Passagen des Romans im sekundären intermedialen Produkt hervorgehoben werden können, so ist dieser dritte Satz aus Henzes Konzert einem hohen Grad zuzuordnen.

\section{$9.3 \quad$ Fazit}

Dieses Kapitel hat im kleinen Rahmen noch einmal gezeigt, was dieser gesamte dritte Teil zu den Figuren aus dem Roman herausstellen sollte: Der Fokus der Untersuchung ist leicht unterschiedlich, wenn man sich auf den Transfer von Romanfiguren in das Medium der Musik konzentriert. Anhand des Satzes von Henze konnte über viele Untersuchungsobjekte dieser Studie reflektiert werden, da der hier behandelte dritte Satz sowohl die Schwerdtfeger-Kapitel und folglich seine Charakterisierung im Roman als auch Leverkühns Violinkonzert transferiert. Im ersten Fall handelt es sich um die Evokation, Simulation oder (Teil-)reproduktion von handlungsbezogenen Passagen oder Figurencharakteristika, die in einem Erzähltext vorwiegend durch telling und/oder showing vonseiten der Erzählinstanz zum Tragen kommen. Im zweiten Fall wird im neuen Medium versucht, das intermediale telling des Romans, also die Beschreibung von Faktur und Wirkung fiktiver Musikstücke, umzusetzen. Die Gegenstände der Untersuchung und folglich die erzähltheoretischen sowie intermedialen Kategorien, mit denen sich die Analyse auseinandersetzt, sind nicht komplett miteinander vergleichbar.

Ebenfalls konnte dieses Kapitel zeigen, dass der Effekt der Verstärkung, den Gess einführt, ${ }^{68}$ analytisch vertieft und erweitert werden kann, indem man ihn qualitativ ausdifferenziert und dementsprechend skalar auffasst. Henze beispielsweise rückt eine Figur aus dem Roman, auf die sonst wenige Beiträge zu Doktor

${ }^{68} \mathrm{Vgl}$. 1.1.5. 
Faustus eingehen, ins Zentrum und erreicht somit einen hohen Verstärkungsgrad. Daran wird noch einmal deutlich, was bereits in der Einleitung dieser Studie angesprochen wurde: Die Auseinandersetzung mit den kompositorischen Reaktionen auf Thomas Manns Roman bewirkt eine neue Lektüre des Werkes, in diesem Fall z. B. wird einer Figur und ihrer Mythisierung mehr Aufmerksamkeit geschenkt. Im nächsten Kapitel soll sich die Untersuchung auf eine*n Akteur*in im Musikbereich, nämlich den Impresario bzw. die Impresaria, konzentrieren, was durch die Analyse des jüdischen Musikagenten Saul Fitelberg möglich ist.

Open Access Dieses Kapitel wird unter der Creative Commons Namensnennung - Nicht kommerziell - Keine Bearbeitung 4.0 International Lizenz (http://creativecommons.org/lic enses/by-nc-nd/4.0/deed.de) veröffentlicht, welche die nicht-kommerzielle Nutzung, Vervielfältigung, Verbreitung und Wiedergabe in jeglichem Medium und Format erlaubt, sofern Sie den/die ursprünglichen Autor(en) und die Quelle ordnungsgemäß nennen, einen Link zur Creative Commons Lizenz beifügen und angeben, ob Änderungen vorgenommen wurden. Die Lizenz gibt Ihnen nicht das Recht, bearbeitete oder sonst wie umgestaltete Fassungen dieses Werkes zu verbreiten oder öffentlich wiederzugeben.

Die in diesem Kapitel enthaltenen Bilder und sonstiges Drittmaterial unterliegen ebenfalls der genannten Creative Commons Lizenz, sofern sich aus der Abbildungslegende nichts anderes ergibt. Sofern das betreffende Material nicht unter der genannten Creative Commons Lizenz steht und die betreffende Handlung nicht nach gesetzlichen Vorschriften erlaubt ist, ist auch für die oben aufgeführten nicht-kommerziellen Weiterverwendungen des Materials die Einwilligung des jeweiligen Rechteinhabers einzuholen.

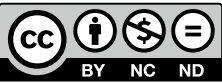

